\title{
Additional effect of etanercept or infliximab on the liver function tests of patients with rheumatoid arthritis: a cohort study
}

This article was published in the following Dove Press journal: Therapeutics and Clinical Risk Management

\author{
Saeed Akhlaghi' \\ Maryam Sahebari ${ }^{2}$ \\ Mahmoud Mahmoodi' \\ Mehdi Yaseri' \\ Mohammad Ali Mansournia' \\ Houshang Rafatpanah ${ }^{3}$ \\ Hojjat Zeraati' \\ 'Department of Epidemiology and \\ Biostatistics, School of Public Health, \\ Tehran University of Medical Sciences, \\ Tehran, Iran; ${ }^{2}$ Rheumatic Diseases \\ Research Center (RDRC), Mashhad \\ University of Medical Sciences, \\ Mashhad, Iran; ${ }^{3}$ Inflammation and \\ Inflammatory Diseases Research \\ Center, Mashhad University of Medical \\ Sciences, Mashhad, Iran
}

Purpose: One of the most important long-term side effects of therapy for rheumatoid arthritis (RA) is the elevation of liver function tests, with earlier studies reporting an elevation of more than $1 \times$ the upper limit of normal $(>1 \times U L N)$. The current study expands the literature by comparing the trends of transaminase changes caused by conventional and biologic diseasemodifying antirheumatic drugs (DMARDs).

Patients and methods: The drug categories examined were methotrexate (MTX) and all other nonbiologic DMARDs. Where RA patients exhibited inadequate response to conventional DMARDs (cDMARDs), we added biologic DMARDs (bDMARDs) to the treatment. We compared the trend of changes in alanine aminotransferase (ALT) and aspartate aminotransferase (AST) in the patients receiving MTX with the trend observed in the patients whose treatment encompassed both bDMARDs and MTX. The comparison was conducted using random intercept models, which are a type of linear mixed effects model.

Results: This work involved 512 RA patients (MTX: 450, MTX + infliximab [INF]: 26, MTX+ etanercept [ETA]: 36), whose ALT and/or AST levels were measured in 1,786 visits (MTX: 1,543, MTX + INF: 107, MTX + ETA: 136). ALT and/or AST elevations greater than $1 \times$ ULN were observed in 344 (19.3\%) visits (MTX: 295 [19.1\%], MTX + INF/ETA: 49 [20.2\%]). In this study, the trends of ALT and AST changes increased when receiving MTX, while the INF/ETA addition decreased these trends. The random intercept models indicated that changes in the mean ALT levels were significantly different over the time for MTX and MTX + INF/ETA groups $(\beta[\mathrm{SE}]=-0.190$ [0.093], $P=0.040)$ but changes in the mean AST levels were nonsignificantly different over the time for such groups $(\beta[\mathrm{SE}]=-0.099$ [0.064], $P=0.120)$.

Conclusion: Despite a higher incidence of elevated transaminases during the use of MTX + INF/ETA, the combination of INF/ETA with MTX reduced transaminase levels and returned ALT levels to normal concentrations.

Keywords: longitudinal, ALT, AST, DMARDs, biologic DMARDs, MTX

\section{Introduction}

Methotrexate (MTX) intervention, as a single-drug treatment or combined with other conventional disease-modifying antirheumatic drugs (cDMARDs), is known as a firstline strategy for patients with newly diagnosed and established rheumatoid arthritis (RA). ${ }^{1,2}$ When patients do not respond appropriately to cDMARDs, biologic DMARDs (bDMARDs) are added to standard treatment. ${ }^{1,2}$ If treatment is effective, these drugs are administered to patients for many years - a situation that highlights the essentiality of providing information on long-term safety. One of the most important side effects of RA therapy is the elevation of transaminase levels. ${ }^{3-16}$ Previous studies reported an
Correspondence: Hojjat Zeraati Department of Epidemiology and Biostatistics, School of Public Health, Tehran University of Medical Sciences, Tehran I4I76I315I, Iran

Tel +982166499713

Fax +982166495936

Email zeraatih@tums.ac.ir 
elevation of liver function tests (LFTs) greater than $1 \times$ the upper limit of normal $(>1 \times \mathrm{ULN}){ }^{6,9,10}$

The current study compared the trends of transaminase changes caused by MTX and its combination with infliximab/ etanercept (INF/ETA). To this end, it was important to take into account the best form of outcomes (qualitative or quantitative) so that a definitive decision can be taken in the shortest possible follow-up duration. When the incidence of elevated liver enzymes in two or more therapeutic groups is compared, an increase in liver enzyme concentration may be observed, but not so much that it can be detected with respect to existing cutoffs. This issue is relevant, especially if follow-up duration is short; such increase may be corrected once it is detected, and drug dosage may be modified or new drugs may be incorporated into treatment. In these conditions, increases in one of the outcomes may be undetected when the categorical form of outcomes is considered. In cases characterized by short follow-up and small sample sizes, discrepancies may be identified through investigations into the quantitative trend of outcomes.

\section{Patients and methods}

\section{Patient population and inclusion criteria}

In this cohort study, analyses were directed toward patients with physician-diagnosed RA based on 1987 American College of Rheumatology Classification Criteria and were referred for treatment at the Rheumatic Diseases Research Center in Mashhad, Iran from October 2008 to March 2016. This study was approved by the ethical committee of School of Public Health, Tehran University of Medical Sciences from Iran (approval no IR.TUMS.SPH.REC.1395.777) and complied with the requirements of the Helsinki Declaration. Written informed consent to participate in the study was obtained from all participants.

A total of 512 RA patients with $3.06 \pm 1.56$ visits to the research center per year were recruited. All the patients received at least 6 months of MTX treatment with varying doses (10-25 mg per week). The conventional treatment for 62 patients was supplemented with INF/ETA because of inadequate response (disease activity score 28 more than 2.5 or the occurrence of laboratory or gastrointestinal complications, after at least 6 months of treatment with MTX or an increased dose of it) to the initial intervention. Among the subjects, 26 were administered MTX + INF, and 36 were prescribed MTX + ETA. All other patients receiving bDMARDs other than INF and ETA were excluded from the analyses. Dose of INF was $3 \mathrm{mg} / \mathrm{kg}$ based on standard protocol, and dose of ETA was $50 \mathrm{mg}$ per week. Indeed according to the guidelines for prescribing MTX and biologic agents, hepatitis profiles were checked and patients with current or past viral hepatitis history were excluded from the study.

\section{Drug exposure definitions and models}

Drug categories included individual MTX (EBEWE, Unterach, Austria) and all other nonbiologic DMARDs, such as sulfasalazine (SSZ [Mehr Darou, Tehran, Iran]) and hydroxychloroquine (HCQ [Rouz Darou, Tehran, Iran]). As previously stated, where the patients exhibited inadequate response to MTX, INF/ETA were added to the treatment regimen. We compared the trend of alanine aminotransferase (ALT) and aspartate aminotransferase (AST) changes in the RA patients receiving MTX with the trend observed among the patients whose treatment was supplemented with INF (Janssen, Leiden, the Netherlands) or ETA (Pfizer, Berlin, Germany).

\section{Outcomes}

The study outcomes of interest were ALT and AST levels, which were evaluated at least two times a year. The ULN cutoffs for ALT and AST concentrations were 41 and $37 \mathrm{U} / \mathrm{L}$ and 31 and $31 \mathrm{U} / \mathrm{L}$ for males and females, respectively. An ALT and/or AST $>1 \times$ ULN was defined as reflecting an elevation in LFT, $>2 \times$ ULN was regarded as indicating abnormality, and $>5 \times$ ULN was considered indicative of hepatotoxicity. ${ }^{17}$ ALT and AST levels were analyzed using a Pars Azmun kit (Pars Azmun, Iran) according to the manufacturer's instructions.

\section{Statistical methods}

Quantitative variables were described as mean \pm SD or median (IQR), and qualitative variables were described in counts and percentages. A chi-squared test was conducted to compare the sex, employment, and rheumatoid factors (RFs) of the MTX, MTX + INF and MTX + ETA groups. Age and disease duration across the groups were compared using analysis of variance and Kruskal-Wallis tests, respectively. Incidence proportions both in terms of visit and period level were presented. All patients experienced MTX treatment period. Sixty-two patients, who did not adequately respond to MTX, experienced MTX + INF or MTX + ETA treatment period too. Incidence rates per 100 person-years were calculated for AST and ALT elevation in each of the treatment groups. The longitudinal effects of drug exposure on ALT and AST were determined via a test of interaction between time and treatment in random intercept models. ${ }^{18,19}$ The R3.3.3 package lme 4 was used to examine mixed effects model, ${ }^{20,21}$ 
and $P$-values were calculated using a likelihood ratio test. Graphs were smoothed via a moving average.

\section{Results}

Table 1 shows the demographic and clinical characteristic of the 512 patients. As indicated by the baseline data, mean age was $47.75 \pm 13.90$ years, and $431(85.2 \%)$ of the patients were women. Among all the patients, 392 (77.6\%) were housekeepers, 78 (15.4\%) were office employees, and 35 (6.9\%) had other types of employment. Additionally, 438 (85.5\%) and 396 (77.3\%) were positive for RFs and anticyclic citrullinated peptid (anti-CCP), respectively. The median (IQR) follow-up per person was 12 (26.75) months.

The chi-squared test showed that the distributions of sex, employment, positive RF, and anti-CCP were nonsignificantly different across the groups $(P=0.561, P=0.461$, $P=0.852, P=0.631$, respectively). Age and duration of follow-up did not significantly differ among the three groups ( $P=0.768$ and $P=0.662$, respectively). The linear mixed effects models showed that changes in the mean MTX doses were significantly different over the time for MTX and MTX + INF/ETA groups $(P<0.001)$ but that no significant changes in HCQ and SSZ were found across the groups ( $P=0.368$ and $P=0.848$, respectively).

Out of the 1,786 visits during which AST and/or ALT levels were measured, 1,543 were related to MTX and 243 were associated with bDMARDs (107 for MTX + INF, 136 for MTX + ETA) (Table 2). The results for MTX group indicated that in 295 (19.1) visits, LFT increased to levels $>1 \times \mathrm{ULN}$; in 47 (3.0) visits, LFT increased to levels $>2 \times \mathrm{ULN}$; in 17 (1.1) visits, LFT increased to levels $>3 \times \mathrm{ULN}$; and in 3 (0.2) visits, LFT elevated to levels $>5 \times$ ULN. In MTX + INF/ETA groups, LFT elevations $>1 \times$ ULN were observed in 17 (14.2) and 32 (23.5) visits, respectively. In terms of period, within 8 years of follow-up, LFT elevations $>1 \times$ ULN and $<2 \times$ ULN were experienced by $126(26.5 \%)$ patients during MTX therapy, 8 (30.8\%) patients during MTX + INF therapy, and $9(25.0 \%)$ patients during MTX + ETA therapy (Table 3 ). LFT elevations $>5 \times$ ULN were observed in 2 $(0.4 \%)$ patients treated with MTX.

The crude model indicated that the mean difference in ALT levels between the onset of INF/ETA treatment and the onset of MTX therapy was $4.627 \mathrm{U} / \mathrm{L}$, which then decreased to $0.091 \mathrm{U} / \mathrm{L}$ after 3 years of bDMARDs treatment (Table 4 ). The mean difference in AST levels between the beginning of INF/ETA intervention and the onset of MTX treatment was $1.428 \mathrm{U} / \mathrm{L}$, which decreased to $-0.444 \mathrm{U} / \mathrm{L}$ after 3 years. The crude model also showed that changes in the mean ALT levels were significantly different over the time for MTX and MTX + INF/ETA groups $(P=0.027)$ but changes in the mean AST levels were nonsignificantly different over the time for such groups $(P=0.105)$. In the MTX dose-adjusted model, the mean difference in ALT levels between the onset of INF/ETA treatment and the onset of MTX intervention was $4.573 \mathrm{U} / \mathrm{L}$, which decreased to $-2.270 \mathrm{U} / \mathrm{L}$ after 3 years. Furthermore, the mean difference in AST levels at the onset

Table I Baseline and disease characteristics in patients receiving MTX, MTX + INF, and MTX + ETA

\begin{tabular}{|c|c|c|c|c|}
\hline & MTX & MTX + INF & MTX + ETA & $P$-value \\
\hline$N$ & 450 & 26 & 36 & \\
\hline Age $\left(\right.$ years) ${ }^{\mathrm{a}}$ & $47.84 \pm 13.83$ & $48.31 \pm 14.25$ & $46.12 \pm 14.87$ & $0.768^{b}$ \\
\hline Female, n (\%) & $376(84.7)$ & $24(92.3)$ & $31(86.1)$ & $0.56 I^{c}$ \\
\hline Body mass index & $23.02 \pm 5.6 I$ & $23.10 \pm 4.61$ & $22.32 \pm 7.07$ & $0.769^{b}$ \\
\hline \multicolumn{5}{|l|}{ Job, n (\%) } \\
\hline House keeper & 345 (77.9) & $20(76.9)$ & $27(75.0)$ & $0.46 I^{c}$ \\
\hline Office employee & $65(14.7)$ & $6(23.1)$ & $7(19.4)$ & \\
\hline Others & $33(7.4)$ & $0(0.0)$ & $2(5.6)$ & \\
\hline $\mathrm{RF}+, \mathrm{n}(\%)$ & $385(85.6)$ & $23(88.5)$ & $30(83.3)$ & $0.852^{c}$ \\
\hline Anti-CCP positive, n (\%) & $35 \mathrm{I}(78.0)$ & $19(73.1)$ & $26(72.2)$ & $0.63 I^{c}$ \\
\hline Visits per year ${ }^{\mathrm{a}}$ & $4.36 \pm 1.41$ & $4.4 I \pm I .83$ & $4.06 \pm 1.69$ & $0.437^{b}$ \\
\hline Follow-up (month) ${ }^{d}$ & II (3I) & $16(29)$ & II (I5.25) & $0.662^{\mathrm{e}}$ \\
\hline Disease duration (years) ${ }^{d}$ & $4.02(1.64)$ & $3.95(1.21)$ & $3.77(1.25)$ & $0.58 \mathrm{I}^{\mathrm{e}}$ \\
\hline $\mathrm{ESR}^{\mathrm{d}}$ & $25.49(25.94)$ & $28.88(34.2)$ & 23.97 (26.59) & $0.898^{e}$ \\
\hline Cardiovascular events, n (\%) & $3(0.7)$ & $0(0.0)$ & $0(0.0)$ & $I^{c}$ \\
\hline Keratoconjunctivitis sicca, n (\%) & I5I (33.6) & $5(19.2)$ & II (30.6) & $0.306^{c}$ \\
\hline Vasculitis, n (\%) & $5(1.1)$ & $0(0.0)$ & $0(0.0)$ & $I^{c}$ \\
\hline Pleuritis, n (\%) & $7(1.6)$ & $0(0.0)$ & $0(0.0)$ & $I^{c}$ \\
\hline
\end{tabular}

Notes: a Mean \pm SD. ${ }^{b}$ ANOVA. ${ }^{c}$ Chi-squared test. ${ }^{\text {Median }}(\mathrm{IQR})$. ${ }^{\mathrm{e}}$ Kruskal-Wallis test.

Abbreviations: ESR, erythrocyte sedimentation rate; ETA, etanercept; INF, infliximab; MTX, methotrexate; RF, rheumatoid factor. 
Table 2 Incidence proportion of elevated liver enzymes within 8 years in visit level in patients receiving MTX, MTX + INF, and MTX + ETA

\begin{tabular}{l|l|l|l}
\hline LFT & MTX, n (\%) & MTX + INF, n (\%) & MTX + ETA, n (\%) \\
\hline Normal & $1,248(80.9)$ & $90(85.8)$ & $104(76.5)$ \\
$>1 \times$ ULN, $<2 \times$ ULN & $248(16.1)$ & $15(12.4)$ & $26(19.1)$ \\
$>2 \times$ ULN, $<3 \times$ ULN & $30(1.9)$ & $2(1.8)$ & $3(2.2)$ \\
$>3 \times$ ULN, $<4 \times$ ULN & $14(0.9)$ & $0(0.0)$ & $3(2.2)$ \\
$>5 \times$ ULN & $3(0.2)$ & $0(0.0)$ & $0(0.0)$ \\
Total & $1,543(100.0)$ & $107(100.0)$ & $136(100.0)$ \\
\hline
\end{tabular}

Abbreviations: ETA, etanercept; INF, infliximab; LFT, liver function test; MTX, methotrexate; ULN, upper limit of normal.

of INF/ETA treatment and the initiation of MTX intervention was $1.319 \mathrm{U} / \mathrm{L}$, which decreased to $-1.633 \mathrm{U} / \mathrm{L}$ in the next 3 years. This model revealed that changes in the mean ALT levels were significantly different over the time for MTX and MTX + INF/ETA groups but AST concentrations were not ( $P=0.040$ and $P=0.120$, respectively).

Table 5 shows that changes in the mean ALT levels were the only significant difference over the time for MTX and MTX + ETA groups. $(P=0.018)$. Mean response profiles of AST were not significantly different for MTX and MTX + ETA groups, and this is true for the mean response profiles of ALT and AST in MTX and MTX + INF groups $(P=0.230, P=0.866$, and $P=0.248$, respectively). The incidence of liver enzyme elevations $>1 \times$ ULN was compared in the studied groups. Despite the decreasing trend of elevated liver enzymes at the time of INF/ETA addition, the interaction term between time and groups (MTX vs MTX+ INF/ETA) was nonsignificant.

In all the models, random intercept terms were statistically significant, indicating that linear mixed effects models were suitable for our data.

\section{Discussion}

Despite the advances achieved in drug production for RA patients, the long-term complications and safety of bDMARDs and their combination with cDMARDs remain controversial issues. Research on these matters can help physicians prescribe medications in monotherapy or in combination therapy in accordance with patients' conditions. In this study, we compared the safety of INF/ETA and MTX on the basis of an appropriate sample size and follow-up duration. The main results indicated that despite the high incidence of elevated liver enzymes during the use of MTX+ INF/ETA, the trend decreased after the addition of the drugs to patients' treatment.

\section{Comparison of trends of liver enzyme concentration}

A trend of increasing liver enzyme concentrations was observed in this study because of the use of MTX, which may lead to liver toxicity and serious complications in long-term use (Figures 1 and 2). A decline in hepatic enzyme concentrations was also observed following the addition of INF/ETA to MTX (Figures 1 and 2). In both the crude and MTX doseadjusted models, MTX + INF/ETA had a significant effect on ALT levels but not on AST levels. In these adjusted models, the mean values of ALT (2.27 U/L) and AST (1.633 U/L) were lower 3 years after the initiation of INF/ETA treatment than at the onset of such intervention.

The increasing trend of liver enzyme concentrations in conventional RA treatment has been documented and agreed in previous studies. For example, in a systematic review that was intended to examine the long-term safety of MTX monotherapy in RA patients, low-dose MTX treatment over 55 months resulted in $20 \%$ of the patients experiencing an elevation in transaminases, $13 \%$ experiencing a transaminase

Table 3 Incidence proportion of elevated liver enzyme within 8 years in period level

\begin{tabular}{|c|c|c|c|}
\hline \multirow[t]{2}{*}{ LFT } & \multicolumn{3}{|l|}{ Period } \\
\hline & MTX, n (\%) & MTX + INF, n (\%) & MTX + ETA, n (\%) \\
\hline Normal & $321(67.6)$ & $16(61.5)$ & $23(62.2)$ \\
\hline$>\mathrm{I} \times \mathrm{ULN},<2 \times \mathrm{ULN}$ & $126(26.5)$ & $8(30.8)$ & $9(25.0)$ \\
\hline$>2 \times$ ULN,$<3 \times$ ULN & $17(3.6)$ & $2(7.7)$ & $3(8.3)$ \\
\hline$>3 \times$ ULN,$<4 \times$ ULN & $9(1.9)$ & $0(0.0)$ & I (2.8) \\
\hline$>5 \times$ ULN & $2(0.4)$ & $0(0.0)$ & $0(0.0)$ \\
\hline Total & $475(100.0)$ & $26(100.0)$ & $36(100.0)$ \\
\hline
\end{tabular}

Abbreviations: ETA, etanercept; INF, infliximab; LFT, liver function test; MTX, methotrexate; ULN, upper limit of normal. 
Table 4 Effect of MTX + INF/ETA on ALT and AST changes unadjusted and adjusted by MTX dose

\begin{tabular}{|c|c|c|c|c|c|c|c|}
\hline \multirow[t]{2}{*}{ Outcome } & \multicolumn{4}{|l|}{ Crude } & \multicolumn{3}{|c|}{ Adjusted by MTX dose } \\
\hline & Effect & Estimate, U/L & $95 \% \mathrm{Cl}$ & $P$-value ${ }^{a}$ & Estimate, U/L & $95 \% \mathrm{Cl}$ & $P$-value ${ }^{a}$ \\
\hline$\overline{\mathrm{ALT}}$ & $\begin{array}{l}\text { Intercept } \\
\text { MTX + INF/ETA } \\
\text { Time } \\
(\text { MTX + INF/ETA }) \times \text { time }\end{array}$ & $\begin{array}{l}21.558 \\
4.627 \\
0.069 \\
-0.195\end{array}$ & $\begin{array}{l}(19.980,23.136) \\
(0.730,8.523) \\
(0.008,0.129) \\
(-0.367,-0.022)\end{array}$ & $\begin{array}{l}<0.001 \\
0.019 \\
0.026 \\
0.027\end{array}$ & $\begin{array}{l}23.470 \\
4.573 \\
-0.0001 \\
-0.190\end{array}$ & $\begin{array}{l}(20.064,26.876) \\
(0.555,8.591) \\
(-0.147,0.146) \\
(-0.372,-0.007)\end{array}$ & $\begin{array}{l}<0.001 \\
0.025 \\
0.981 \\
0.040\end{array}$ \\
\hline$\overline{\mathrm{AST}}$ & $\begin{array}{l}\text { Intercept } \\
\text { MTX + INF/ETA } \\
\text { Time } \\
(\text { MTX + INF/ETA }) \times \text { time }\end{array}$ & $\begin{array}{l}21.145 \\
1.428 \\
0.047 \\
-0.099\end{array}$ & $\begin{array}{l}(20.133,22.157) \\
(-1.221,4.077) \\
(0.007,0.086) \\
(-0.218,0.020)\end{array}$ & $\begin{array}{l}<0.001 \\
0.291 \\
0.022 \\
0.105\end{array}$ & $\begin{array}{l}22.576 \\
1.319 \\
0.017 \\
-0.099\end{array}$ & $\begin{array}{l}(20.290,24.862) \\
(-1.423,4.061) \\
(-0.082,0.116) \\
(-0.224,0.026)\end{array}$ & $\begin{array}{l}<0.001 \\
0.344 \\
0.732 \\
0.120\end{array}$ \\
\hline
\end{tabular}

Note: aLikelihood ratio test.

Abbreviations: ALT, alanine aminotransferase; AST, aspartate aminotransferase; ETA, etanercept; INF, infliximab; MTX, methotrexate.

increase of more than twice the ULN, and $3.7 \%$ discontinuing MTX due to liver toxicity. ${ }^{4}$

The results of the present research align with those of several other studies that found the combination of cDMARDs and bDMARDs in RA treatment to result in an increase in the efficacy and safety of the drugs., ${ }^{3,1522-26}$ A study on the safety of cDMARDs and bDMARDs showed that the side effects of RA monotherapy are nonsignificantly different, whereas the combination of cDMARDs and bDMARDs, compared with the use of cDMARDs alone, presents a lower risk of drug discontinuation because of side effects (HR $=0.24 ; 95 \% \mathrm{CI}=0.09-0.6) .{ }^{3}$ In another review of RA patients, bDMARDs were found to be exactly equivalent to a concomitant treatment involving MTX and bDMARDs, albeit this claim has not been verified. ${ }^{27}$

In a review intended to evaluate the safety and efficacy of ETA and INF on RA patients, the researchers reported that elevated liver enzymes during treatment with ETA decrease to levels lower than those observed under MTX treatment. In addition, the side effects of INF nonsignificantly differ from those of MTX. ${ }^{28}$ Another review article on the safety of bDMARDs found abnormal LFTs and rare hepatotoxicity under treatment with tumor necrosis factor (TNF) inhibitors. ${ }^{7}$ A prospective cohort study that assessed the risk of elevated liver enzymes revealed that the rates of liver enzyme increase that were greater than $1 \times$ ULN per visit were $4.9 \%$ for antiTNF, $4.8 \%$ for ETA, and $6.7 \%$ for INF. Abnormalities of more than $2 \times$ ULN occurred at a rate of $0.6 \%$ for ETA and at a rate of $0.9 \%$ for INF. ${ }^{17}$ The odds of observing elevated liver enzymes in the group that involved the concomitant use of traditional drugs and bDMARDs compared with the group treated with only bDMARDs were $1.00(95 \% \mathrm{CI}=0.83-1.21)$ for ETA and 1.58 (95\% CI $=1.35-1.86)$ for INF. ${ }^{17}$ These contradictions are attributed to the three fundamental differences in the design of Sokolove et al's ${ }^{17}$ study and our work. First, our study quantitatively analyzed ALT and AST concentrations, driving us to make decisions about the effectiveness of bDMARDs in a shorter time frame. In the study of Sokolove et al, the incidence of elevated liver enzymes was compared in therapeutic groups. ${ }^{17}$ Given the low incidence rate of these events, a study should be long enough to enable definitive decisions on the effects of bDMARDs. A longer study will

Table 5 Effect of ETA + MTX and INF + MTX on ALT and AST changes adjusted by MTX dose

\begin{tabular}{|c|c|c|c|c|c|c|}
\hline \multirow[t]{3}{*}{ Effect } & \multicolumn{6}{|l|}{ Outcome } \\
\hline & \multicolumn{3}{|l|}{ ALT } & \multicolumn{3}{|l|}{ AST } \\
\hline & Estimate $^{\mathrm{a}}, \mathrm{U} / \mathrm{L}$ & $95 \% \mathrm{Cl}$ & $P$-value ${ }^{b}$ & Estimate $^{a}, \mathrm{U} / \mathrm{L}$ & $95 \% \mathrm{Cl}$ & $P$-value ${ }^{\mathrm{b}}$ \\
\hline Intercept & 23.670 & $(20.122,27.218)$ & $<0.001$ & 22.751 & $(20.375,25.127)$ & $<0.001$ \\
\hline $\mathrm{ETA}+\mathrm{MTX}$ & 8.228 & $(3.053,13.402)$ & 0.001 & 2.676 & $(-0.832,6.184)$ & 0.134 \\
\hline Time & 0.001 & $(-0.151,0.149)$ & 0.985 & 0.017 & $(-0.085,0.119)$ & 0.739 \\
\hline$(\mathrm{ETA}+\mathrm{MTX}) \times$ time & -0.252 & $(-0.453,-0.050)$ & 0.014 & -0.106 & $(-0.243,0.031)$ & 0.130 \\
\hline Intercept & 23.009 & $(19.421,26.597)$ & $<0.001$ & 22.276 & $(19.838,24.714)$ & $<0.001$ \\
\hline INF + MTX & -0.143 & $(-6.505,6.219)$ & 0.963 & 0.748 & $(-3.697,5.193)$ & $0.74 I$ \\
\hline Time & 0.018 & $(-0.135,0.17 \mid)$ & 0.811 & 0.034 & $(-0.071,0.140)$ & 0.527 \\
\hline$(\mathrm{INF}+\mathrm{MTX}) \times$ time & -0.011 & $(-0.4|2,0.39|)$ & 0.954 & -0.113 & $(-0.399,0.173)$ & 0.435 \\
\hline
\end{tabular}

Notes: adjusted by MTX dose. 'bikelihood ratio test.

Abbreviations: ALT, alanine aminotransferase; AST, aspartate aminotransferase; ETA, etanercept; INF, infliximab; MTX, methotrexate. 


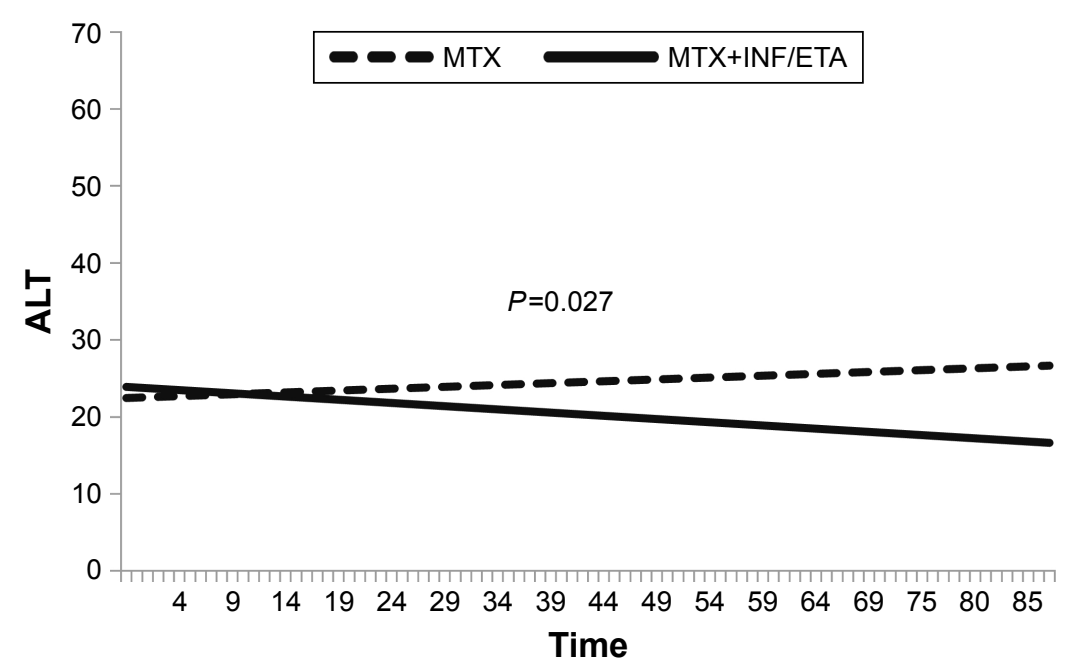

Figure I Mean trend of ALT in MTX therapy period vs period of adding ETA or INF.

Abbreviations: ALT, alanine aminotransferase; ETA, etanercept; INF, infliximab; MTX, methotrexate.

lead to more complicated confounders, some of which may be unmeasurable. ${ }^{29}$ Second, an independent bDMARDsreceiving group was not included in our work, thereby leading to regression-to-the-mean bias. Third, in the study of Sokolove et al, the patients were censored after their first LFT elevation, ${ }^{17}$ and the authors disregarded recurrent LFT elevations. In this case, the incidence rate of LFT elevation may have been underestimated.

\section{Comparison of incidence proportions and incidence rates of elevated liver enzyme concentration}

Table 2 shows that the incidence proportion of elevated transaminases $>1 \times$ ULN for MTX, MTX + INF,
MTX + ETA were 19.1\%, 14.2\%, and 23.5\%, respectively. The reported events in total indicated that the initiation of treatment with INF/ETA increased liver enzyme concentrations. Approximately $32.4 \%$ of the patients at the time of MTX intervention and $34.7 \%$ of the patients at the time of MTX + INF/ETA intervention experienced at least one event of transaminase increase to levels $>1 \times$ ULN (Table 3 ). Furthermore, considering each person's follow-up time, the incidence rates of liver enzyme elevations $>1 \times$ ULN at the period of MTX, MTX + INF, and MTX + ETA interventions were $37.2,28.4$, and 39.8 in 100 patient-years, respectively.

A study that evaluated the safety of bDMARDs in RA patients demonstrated that increased liver enzymes may occur

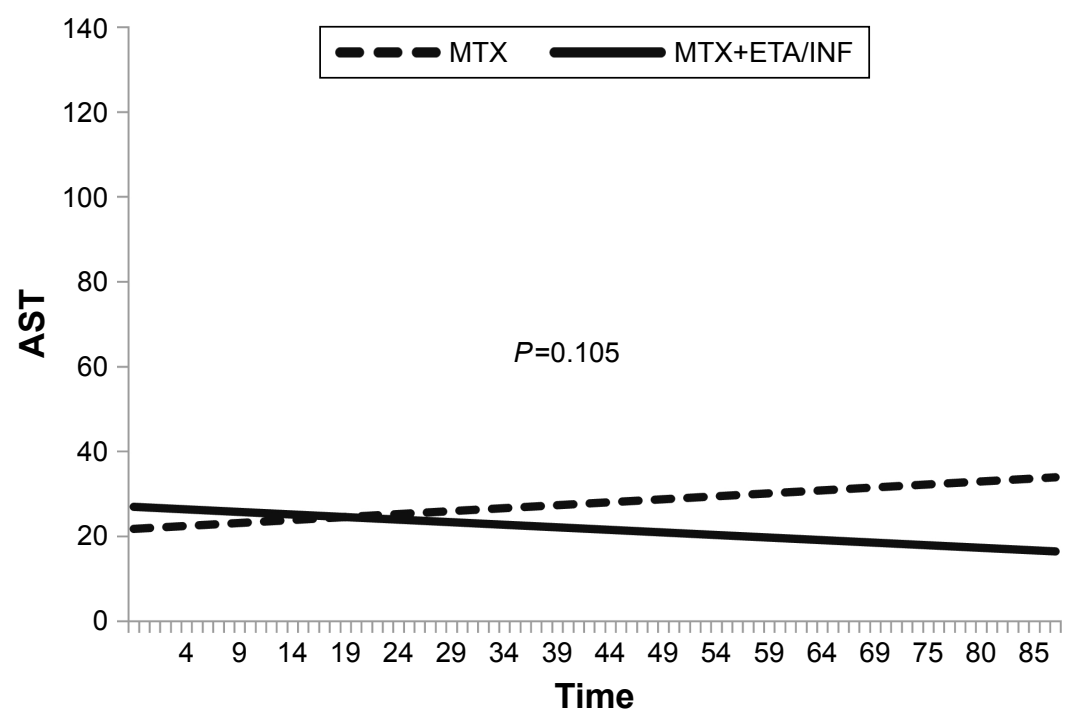

Figure 2 Mean trend of AST in MTX therapy period vs period of adding ETA or INF.

Abbreviations: AST, aspartate aminotransferase; ETA, etanercept; INF, infliximab; MTX, methotrexate. 
during treatment with bDMARDs; this effect is often mild to moderate, transient and without clinical symptoms, and is severe in less than $0.9 \%$ of patients $(>5 \times \mathrm{ULN}) .{ }^{10} \mathrm{In}$ a prospective cohort study, the researchers assessed the effects of bDMARDs monotherapy and combined bDMARDs and cDMARDs on the basis of 22,522 visits with 6,861 patients. Increased liver enzyme concentrations following TNF inhibitor usage occurred in $5.9 \%$ of the visits. ${ }^{17}$ In a historical cohort study, 949 cases of side effects occurred in 419 RA patients (32.8\% side effects per 100 patient-years). The number of adverse reactions reported for INF, ETA, and MTX were $13.3,17.6$, and 6.6 in 100 patient-years, respectively. The most frequently reported side effects were those related to elevated transaminase and dysphagia. The results, which are incompatible with our findings, also indicated that RA treatments lead to side effects, especially in patients receiving bDMARDs. ${ }^{9}$ The inconsistency of between our results and those of previous studies may be ascribed to the difference in follow-up periods. Additional contributors to this difference include the time at which liver enzyme concentrations were measured, drug doses, drug interactions, and the lack of regular follow-ups.

In the present study, we demonstrated that combination of MTX and INF/ETA reduces the levels of transaminases in RA patients. It has been shown that immune response in RA patients is associated with shift toward Th1 cytokines such as IFN- $\gamma$ and TNF- $\alpha{ }^{30}$ Studies have shown that MTX induces Th2 cytokine such as IL-10 and inhibits Th1 profile in RA patients. ${ }^{31}$ It seems that suppression of Th1 cytokine by MTX + INF or MTX + ETA blocks macrophage activation thereby resulting in liver protection.

The limitations of the current research are worth noting. First, the patients may not have disclosed all the medications that they were taking. Nevertheless, the distributions of these drugs in the treatment groups were expected to be nonsignificantly different. Second, given that time-varying confounding variables were not measured, adjusting for the effects of these variables was not possible. ${ }^{29}$ Third, a quantitative study on transaminase measurements was not available for comparisons. Avoiding regression-to-the-mean bias necessitates that a parallel group of patients who have been receiving bDMARDs for disease onset be included in analysis. Fourth, contrary to our expectations, previous longitudinal studies did not use the incidence rate index to describe data, thus preventing a clear comparison of our work with previous studies.

The rate of liver toxicity in this study was very low. With an increase in follow-up duration and sample size, especially with respect to bDMARDs intervention, outcomes such as liver toxicity can be evaluated and compared across therapeutic groups. We also suggest that liver enzyme concentrations be compared quantitatively in parallel groups to reduce regression-to-the-mean bias.

\section{Acknowledgments}

This study was part of a $\mathrm{PhD}$ thesis supported by Tehran University of Medical Sciences. We would like to show my warm thanks to Dr Amin Saeidinia who constructed the framework of the discussion.

\section{Author contributions}

MS contributed to providing the data, conception and design of the work, and revision of final article. HZ and MM supervised the whole work, and MY and MAM had a big contribution in finding the right model. SA collected and analyzed the data, interpreted the results, and had a major contribution in writing the manuscript. All authors contributed toward data analysis, drafting and critically revising the paper and agree to be accountable for all aspects of the work.

\section{Disclosure}

The author reports no conflicts of interest in this work.

\section{References}

1. Smolen JS, Landewé R, Breedveld FC, et al. EULAR recommendations for the management of rheumatoid arthritis with synthetic and biological disease-modifying antirheumatic drugs: 2013 update. Ann Rheum Dis. 2014;73(3):492-509.

2. Machado P, Castrejon I, Katchamart W, et al. Multinational evidencebased recommendations on how to investigate and follow-up undifferentiated peripheral inflammatory arthritis: integrating systematic literature research and expert opinion of a broad international panel of rheumatologists in the 3E Initiative. Ann Rheum Dis. 2011;70(1):15-24.

3. Abásolo L, León L, Rodríguez-Rodriguez L, et al. Safety of diseasemodifying antirheumatic drugs and biologic agents for rheumatoid arthritis patients in real-life conditions. Semin Arthritis Rheum. 2015; 44(5):506-513.

4. Salliot C, van der Heijde D. Long-term safety of methotrexate monotherapy in patients with rheumatoid arthritis: a systematic literature research. Ann Rheum Dis. 2009;68(7):1100-1104.

5. Kremer JM, Phelps CT. Long-term prospective study of the use of methotrexate in the treatment of rheumatoid arthritis. Update after a mean of 90 months. Arthritis Rheum. 1992;35(2):138-145.

6. Visser K, van der Heijde DM. Risk and management of liver toxicity during methotrexate treatment in rheumatoid and psoriatic arthritis: a systematic review of the literature. Clin Exp Rheumatol. 2009;27(6): $1017-1025$.

7. Woodrick RS, Ruderman EM. Safety of biologic therapy in rheumatoid arthritis. Nat Rev Rheumatol. 2011;7(11):639-652.

8. Ruderman EM. Overview of safety of non-biologic and biologic DMARDs. Rheumatology. 2012;51(SUPPL 6):37-43

9. Machado-Alba JE, Ruiz AF, Machado-Duque ME. Adverse drug reactions associated with the use of disease-modifying anti-rheumatic drugs in patients with rheumatoid arthritis. Rev Panam Salud Publica. 2014;36(6):396-401.

10. Rubbert-Roth A. Assessing the safety of biologic agents in patients with rheumatoid arthritis. Rheumatology. 2012;51(suppl 5):v38-v47. 
11. Storage SS, Agrawal H, Furst DE. Description of the efficacy and safety of three new biologics in the treatment of rheumatoid arthritis. Korean J Intern Med. 2010;25(1):1-17.

12. Sakai R, Cho S-K, Nanki T, et al. Head-to-head comparison of the safety of tocilizumab and tumor necrosis factor inhibitors in rheumatoid arthritis patients (RA) in clinical practice: results from the registry of Japanese RA patients on biologics for long-term safety (REAL) registry. Arthritis Res Ther. 2015;17(1):1-10.

13. Aithal GP. Hepatotoxicity related to antirheumatic drugs. Nat Rev Rheumatol. 2011;7(3):139-150.

14. Curtis JR, Beukelman T, Onofrei A, et al. Elevated liver enzyme tests among patients with rheumatoid arthritis or psoriatic arthritis treated with methotrexate and/or leflunomide. Ann Rheum Dis. 2010; 69(01):43-47.

15. Anelli MG, Scioscia C, Grattagliano I, Lapadula G. Old and new antirheumatic drugs and the risk of hepatotoxicity. Ther Drug Monit. 2012;34(6):622-628.

16. Tobon GJ, Cañas C, Jaller JJ, Restrepo JC, Anaya JM. Serious liver disease induced by infliximab. Clin Rheumatol. 2007;26(4):578-581.

17. Sokolove J, Strand V, Greenberg JD, et al. Risk of elevated liver enzymes associated with TNF inhibitor utilisation in patients with rheumatoid arthritis. Ann Rheum Dis. 2010;69(9):1612-1617.

18. Fitzmaurice G, Laird N, Ware J. Applied Longitudinal Analysis. 1st ed. Hoboken, New Jersey: Wiley, John \& Sons; 2004.

19. Agresti A. Categorical Data Analysis. 3rd ed. Wiley: John \& Sons; 2012.

20. R Development Core Team. R: A language and environment for statistical computing, $R$ Foundation for Statistical Computing. Vienna, Austria; 2017.

21. Bates D, Mächler M, Bolker B, Walker S. Fitting linear mixed-effects models using lme4. J Stat Softw. 2015;67(1):1-48.

22. Romão VC, Lima A, Bernardes M, Canhão H, Fonseca JE. Three decades of low-dose methotrexate in rheumatoid arthritis: can we predict toxicity? Immunol Res. 2014;60(2-3):289-310.
23. Kameda H, Kanbe K, Sato E, et al. Continuation of methotrexate resulted in better clinical and radiographic outcomes than discontinuation upon starting etanercept in patients with rheumatoid arthritis: 52-week results from the JESMR study. J Rheumatol. 2011;38(8):1585-1592.

24. Kameda H, Ueki Y, Saito K, et al. Etanercept (ETN) with methotrexate (MTX) is better than ETN monotherapy in patients with active rheumatoid arthritis despite MTX therapy: a randomized trial. Mod Rheumatol. 2010;20(6):531-538.

25. Emery P, Breedveld FC, Hall S, et al. Comparison of methotrexate monotherapy with a combination of methotrexate and etanercept in active, early, moderate to severe rheumatoid arthritis (COMET): a randomised, double-blind, parallel treatment trial. Lancet. 2008;372(9636): 375-382.

26. Garrison L, Mcdonnell ND. Etanercept: therapeutic use in patients with rheumatoid arthritis. Ann Rheum Dis. 1999;58(Supplement 1):i65-i69.

27. Emery P, Sebba A, Huizinga TW. Biologic and oral disease-modifying antirheumatic drug monotherapy in rheumatoid arthritis. Ann Rheum Dis. 2013;72(12):1897-1904.

28. Mikuls TR, Moreland LW. TNF blockade in the treatment of rheumatoid arthritis: infliximab versus etanercept. Expert Opin Pharmacother. 2001;2(1):75-84.

29. Mansournia MA, Etminan M, Danaei G, Kaufman JS, Collins G. Handling time varying confounding in observational research. $B M J$. 2017;359(October):j4587.

30. He C, Feng P, Su H, Gu A, Yan Z, Zhu X. Disrupted Th1/Th2 balance in patients with rheumatoid arthritis (RA). Int J Clin Exp Pathol. 2017; 10(2):1233-1242.

31. Herman S, Zurgil N, Langevitz P, Ehrenfeld M, Deutsch M. Methotrexate selectively modulates $\mathrm{TH} 1 / \mathrm{TH} 2$ balance in active rheumatoid arthritis patients. Clin Exp Rheumatol. 2008;26(2):317-323.
Therapeutics and Clinical Risk Management

\section{Publish your work in this journal}

Therapeutics and Clinical Risk Management is an international, peerreviewed journal of clinical therapeutics and risk management, focusing on concise rapid reporting of clinical studies in all therapeutic areas, outcomes, safety, and programs for the effective, safe, and sustained use of medicines. This journal is indexed on PubMed Central, CAS,

\section{Dovepress}

EMBase, Scopus and the Elsevier Bibliographic databases. The manuscript management system is completely online and includes a very quick and fair peer-review system, which is all easy to use. Visit http://www.dovepress.com/testimonials.php to read real quotes from published authors. 